\title{
Asymptotic Estimates of the Solution of a Restoration Problem with an Initial Jump
}

\author{
Duisebek Nurgabyl \\ Institute of Postgraduate Education and Staff Retraining, Zhetysu State University Named after I. Zhansugurov, \\ 187A Zhansugurov Street, Taldykorgan 040000, Kazakhstan
}

Correspondence should be addressed to Duisebek Nurgabyl; kebek.kz@mail.ru

Received 18 September 2013; Accepted 20 January 2014; Published 27 April 2014

Academic Editor: Debasish Roy

Copyright (c) 2014 Duisebek Nurgabyl. This is an open access article distributed under the Creative Commons Attribution License, which permits unrestricted use, distribution, and reproduction in any medium, provided the original work is properly cited.

\begin{abstract}
The asymptotic behavior of the solution of the singularly perturbed boundary value problem $L_{\varepsilon} y=h(t) \lambda, L_{i} y+\sigma_{i} \lambda=a_{i}, i=\overline{1, n+1}$ is examined. The derivations prove that a unique pair $(\tilde{y}(t, \tilde{\lambda}(\varepsilon), \varepsilon), \tilde{\lambda}(\varepsilon))$ exists, in which components $y(t, \tilde{\lambda}(\varepsilon), \varepsilon)$ and $\tilde{\lambda}(\varepsilon)$ satisfy the equation $L_{\varepsilon} y=h(t) \lambda$ and boundary value conditions $L_{i} y+\sigma_{i} \lambda=a_{i}, i=\overline{1, n+1}$. The issues of limit transfer of the perturbed problem solution to the unperturbed problem solution as a small parameter approaches zero and the existence of the initial jump phenomenon are studied. This research is conducted in two stages. In the first stage, the Cauchy function and boundary functions are introduced. Then, on the basis of the introduced Cauchy function and boundary functions, the solution of the restoration problem $L_{\varepsilon} y=h(t) \lambda, L_{i} y+\sigma_{i} \lambda=a_{i}, i=\overline{1, n+1}$ is obtained from the position of the singularly perturbed problem with the initial jump. Through this process, the formula of the initial jump and the asymptotic estimates of the solution of the considered boundary value problem are identified.
\end{abstract}

\section{Introduction}

One of the fundamental theorems of singular perturbations theories is Tikhonov's theorem $[1,2]$ on the limit transition that establishes the limit equations, expressing the relations between the solutions of a degenerate problem and an original singularly perturbed initial problem, and this theorem allows us to obtain the leading member of asymptotics.

For a wide class of the singularly perturbed problems, effective asymptotic methods were developed, allowing for the development of uniform approximations with any level of precision. The methods of Višik and Lyusternik [3] and Vasilyeva [4] were the first methods to be developed, which are called methods of boundary functions. Butuzov [5] developed a method of angular boundary functions, which was a significant development for the boundary functions method.

Each of these methods has a certain area of applicability; that is, they are successful in solving some problems and are invalid when solving other problems. For example, there were some fundamental difficulties in the realization of the boundary functions method in problems with initial jumps. The beginning of the mathematical solution of the initial jump phenomenon was considered in the studies of Višik and Lyusternik [6] and Kasymov [7], in which the method of zone integration for nonlinear singularly perturbed initial tasks with unbounded initial data when a small parameter approaches zero. The research efforts of Višik, Lyusternik, and Kasymov were continued in [8,9] and other studies.

Simultaneously, there were problems in practice that extended beyond the scope of traditional research, in which ready-made asymptotic methods were inapplicable and required modification or generalization. For example, in the study of Neǐmark and Smirnova [10], a new rationale of the physical and mathematical nature of the Painleve paradox was introduced, which enriched the possible types of movements and dynamics of the system as a whole. At this point, we saw the races and contrasting structures. Butuzov and Vasiliyeva studied mathematical solutions of the question of contrast structures [11], and the phenomenon of the initial jump requires additional mathematical research. 
Boundary value problems for ordinary differential equations containing parameters in the right-hand side and in the boundary conditions were examined [12,13]. In these studies, a restoration problem of the right-hand side of the differential equations and boundary conditions is solved with the wellknown structure of the differential equation and additional information.

The following natural generalization in this direction is to study the solutions of singularly perturbed boundary value problems with an additional parameter having the initial jump phenomenon. Such a study has not yet been reported. This study considers such problems. It studies the problems of solution building, restoring the right-hand side of the equation and boundary conditions of the perturbed problem, the limit solution passing of the perturbed problem solution to the unperturbed problem solution.

\section{Statement of the Problem}

Let $R=(-\infty, \infty), T=[0,1]$, where $t$ belongs to $T=[0,1]$. We consider

$$
P=\left(\begin{array}{cccccccc}
\alpha_{10} & \alpha_{11} & \ldots & \alpha_{1 m_{1}} & \beta_{10} & \beta_{11} & \ldots & \beta_{1 r_{1}} \\
\alpha_{20} & \alpha_{21} & \ldots & \alpha_{2 m_{2}} & \beta_{20} & \beta_{21} & \ldots & \beta_{2 r_{2}} \\
\ldots & \ldots & & \ldots & \ldots & \ldots & & \ldots \\
\alpha_{n+1,0} & \alpha_{n+1,1} & \ldots & \alpha_{n+1, m_{n+1},} & \beta_{n+1,0} & \beta_{n+1,1} & \ldots & \beta_{n+1, r_{n+1},}
\end{array}\right)
$$

have a rank equal to $n$, where $n$ is the maximum number of linearly independent rows of matrix $P$. To the certainty, let the first $n$ rows of the matrix be linearly independent. Then, the linear forms $L_{i} y, i=\overline{1, n}$, as the functions of $y^{(j)}(0, \varepsilon), j=$ $\overline{0, m_{i}}$ and $y^{(j)}(1, \varepsilon), j=\overline{0, r_{i}}$ are linearly independent of each other.

Consider that

(a) $A_{i}(t) \in C^{n}(T), i=\overline{1, n}, h(t) \in C^{1}(T)$,

(b) a constant $\gamma$ is independent of $\varepsilon$ and is

$$
A_{1}(t) \geq \gamma>0, \quad 0 \leq t \leq 1,
$$

(c) inequalities

$$
\begin{gathered}
\alpha_{1 m_{1}} \neq 0, \quad n \geq 3, \\
n-1>m_{1}>m_{2} \geq \cdots \geq m_{n}, \\
n-1>r_{i}, \quad i=\overline{1, n}, \quad \bar{J}_{01} \neq 0
\end{gathered}
$$

are valid, where $\bar{J}_{01}$ is a determinant of $(n-1)$ th order, which is obtained from the rectangular matrix $L_{i} y_{j 0}(i=1, \ldots, n, j=$ $1, \ldots, n-1)$ as follows:

$$
\bar{J}=\left(\begin{array}{ccc}
L_{1} y_{10} & \cdots & L_{1} y_{n-1,0} \\
\cdots & \cdots & \cdots \\
L_{n} y_{10} & \cdots & L_{n} y_{n-1,0}
\end{array}\right)
$$

$$
L_{\varepsilon} y \equiv \varepsilon y^{(n)}+A_{1}(t) y^{(n-1)}+\cdots+A_{n}(t) y=h(t) \lambda
$$

with nonseparated boundary conditions

$$
L_{i} y+\sigma_{i} \lambda=a_{i}, \quad i=\overline{1, n+1},
$$

where

$$
L_{i} y \equiv \sum_{j=0}^{m_{i}} \alpha_{i j} y^{(j)}(0, \varepsilon)+\sum_{j=0}^{r_{i}} \beta_{i j} y^{(j)}(1, \varepsilon),
$$

$\varepsilon>0$ is a small parameter, $\lambda$ is an unknown parameter, $\alpha_{i j}, \beta_{i j}, a_{i}, \sigma_{i} \in R$ are known constants, and $m_{i}, r_{i}=$ fix $\epsilon$ $\{0,1, \ldots, n-1\}$. Let the matrix

by deleting the first line, and $y_{i 0}(t), i=\overline{1, n-1}$ is the fundamental system of the solutions of the following homogeneous unperturbed (degenerate) equation:

$$
L_{0} y_{0} \equiv A_{1}(t) y_{0}^{(n-1)}+\cdots+A_{n}(t) y_{0}=0 .
$$

Corresponding to (1),

(d) $\bar{D}=\bar{D}_{n+1}-\sum_{i=2}^{n} \bar{D}_{i} L_{n+1} \bar{J}_{i-1,1}(t) / \bar{J}_{01} \neq 0$ is valid,

where $\bar{D}_{i}=\sigma_{i}+\sum_{j=0}^{r_{i}} \beta_{i j} \int_{0}^{1}\left(h(s) \bar{W}_{t}^{(q)}(1, s) / A_{1}(s) \bar{W}(s)\right) d s(i=$ $\overline{1, n+1}), \bar{J}_{i-1,1}(t)$ is the $(n-1)$ th-order determinant obtained from $\bar{J}_{01}$ by replacing the $(i-1)$ th row with the $y_{10}(t), y_{20}(t), \ldots, y_{n-1,0}(t)$ row, and $\bar{W}(s)$ is the Wronskian of the solution system $y_{i 0}(s), i=\overline{1, n-1}, \bar{W}_{t}^{(q)}(t, s)$ and the $(n-1)$ th-order determinant obtained from $\bar{W}(s)$ by replacing the $(i-1)$ th row with the $y_{10}^{(q)}(t), \ldots, y_{n-1,0}^{(q)}(t)$ row.

(e) Consider

$$
\begin{aligned}
& \sum_{i=1}^{n}(-1)^{1+i} \bar{J}_{0 i} \\
& \quad \times\left(a_{i}-\lambda_{0} \sigma_{i}-\lambda_{0} \sum_{j=0}^{r_{i}} \beta_{i j} \int_{0}^{1} \frac{h(s)}{A_{1}(s)} \frac{\bar{W}_{t}^{(q)}(1, s)}{\bar{W}(s)} d s\right) \neq 0,
\end{aligned}
$$


where $\bar{J}_{0 i}$ is the $(n-1)$ th-order determinant, which originates from $\bar{J}$ by deleting the $i$ th row, and $\lambda_{0}=(1 / \bar{D})\left(a_{n+1}-\right.$ $\left.\sum_{i=1}^{n} a_{i} L_{n+1} \bar{J}_{i-1,1}(t) / \bar{J}_{01}\right)$.

The problem lies in determining the pair $(y(t, \tilde{\lambda}(\varepsilon), \varepsilon), \widetilde{\lambda}(\varepsilon))$, where $y(t, \tilde{\lambda}(\varepsilon), \varepsilon)$ and $\tilde{\lambda}(\varepsilon)$ satisfy (1) and the boundary conditions in (2), in building the asymptotic estimates of (1) and (2) problem solutions and in the study of the initial jump phenomenon.

The following study is conducted according to a specific rule. In the first stage, we build the recovery problem solutions (1) and (2) from the position of a singularly perturbed problem with the initial jump on the basis of initial and boundary functions. In the next stage, we study the asymptotic behavior of the boundary value problem solutions (1) and (2).

\section{Fundamental Solution System}

Along with (1), we consider the following corresponding homogeneous perturbed equation:

$$
L_{\varepsilon} y=0
$$

We seek a fundamental solution system of (10) in the following form:

$$
\begin{gathered}
y_{i}(t, \varepsilon)=y_{i 0}+\varepsilon y_{i 1}+\varepsilon^{2} y_{i 2}+\cdots, \quad i=\overline{1, n-1} \\
y_{n}(t, \varepsilon)=\exp \left(\frac{1}{\varepsilon} \int_{0}^{t} \mu(x) d x\right)\left(y_{n 0}+\varepsilon y_{n 1}+\varepsilon^{2} y_{n 2}+\cdots\right),
\end{gathered}
$$

where $y_{i j}(t)$ represents unknown functions to be found and $\mu(t)=-A_{1}(t)$.

By substituting (11) into (10) and by matching the coefficients of like powers of $\varepsilon$ on both sides of the resulting relation, we obtain a sequence of equations for all terms in expansion (11). For our aim, however, it suffices to consider the zero approximation. So, for the zero approximation (for $\left.y_{i 0}(t), i=\overline{1, n-1}\right)$, we have the following problems:

$$
\begin{gathered}
L_{0} y_{i 0}=A_{1}(t) y_{i 0}^{(n-1)}+\cdots+A_{n}(t) y_{i 0}=0, \quad i=\overline{1, n-1}, \\
y_{i 0}^{(j)}(0)=1, \quad j=i-1, \quad y_{i 0}^{(j)}(0)=0, \quad j \neq i-1, \\
j=\overline{0, n-2}, i=\overline{1, n-1}, \\
A_{1}(t) y_{n 0}^{\prime}(t)+\left[(n-1) A_{1}^{\prime}(t)-A_{2}(t)\right] y_{n 0}(t)=0, \\
y_{n 0}(0)=1 .
\end{gathered}
$$

Unique solutions exist for the problems (12) on the interval $0 \leq t \leq 1$, and they form the fundamental solution system $y_{i 0}(t), i=\overline{1, n-1}$ for the following homogeneous equation:

$$
L_{0} y_{0} \equiv A_{1}(t) y_{0}^{(n-1)}+\cdots+A_{n}(t) y_{0}=0
$$

where $y_{n 0}(t)$ is presented in the following form:

$$
y_{n 0}(t)=\left(\frac{A_{1}(0)}{A_{1}(t)}\right)^{n-1} \exp \left(\int_{0}^{t}\left(\frac{A_{2}(x)}{A_{1}(x)}\right) d x\right) .
$$

Lemma 1. Let conditions ( $a$ ) and (b) be satisfied. Then, the fundamental solution system $y_{i}(t, \varepsilon), i=\overline{1, n}$ of the singularly perturbed equation (10) permits the following asymptotic representations:

$$
\begin{aligned}
y_{i}^{(q)}(t, \varepsilon)= & y_{i 0}^{(q)}(t)+O(\varepsilon), \quad i=\overline{1, n-1}, q=\overline{0, n-1}, \\
y_{n}^{(q)}(t, \varepsilon)= & \frac{1}{\varepsilon^{q}} \exp \left(\frac{1}{\varepsilon} \int_{0}^{t} \mu(x) d x\right) \\
& \cdot y_{n 0}(t) \mu^{q}(t)(1+O(\varepsilon)), \quad q=\overline{0, n-1},
\end{aligned}
$$

as $\varepsilon \rightarrow 0$.

The proof of the lemma is readily obtained from the wellknown theorems of Schlesinger [14] and Birkhoff [15] (e.g., see $[16])$.

Let us introduce the Wronskian determinant $W(t, \varepsilon)$ for the fundamental solution system $y_{i}(t, \varepsilon), \quad i=\overline{1, n}$ of (9) and expand it in entries of the $n$th column:

$$
\begin{aligned}
W(t, \varepsilon)= & (-1)^{1+n} y_{n}(t, \varepsilon) W_{1}(t, \varepsilon)+\cdots \\
& +(-1)^{2 n} y_{n}^{(n-1)}(t, \varepsilon) W_{n}(t, \varepsilon),
\end{aligned}
$$

where $W_{i}(t, \varepsilon), i=\overline{1, n}$ is $(n-1)$ th order.

With regard to (15), we obtain

$$
W_{i}(t, \varepsilon)=W_{i 0}(t)+O(\varepsilon), \quad i=\overline{1, n},
$$

for sufficiently small $\varepsilon$ for $W_{i}(t, \varepsilon)$, where $W_{i 0}(t)$ is obtained from the rectangular matrix $\left(y_{j 0}^{(i-1)}(t)\right)(i=1, \ldots, n, j=$ $1, \ldots, n-1)$ by deleting the $i$ th row. In particular, the determinant $W_{n 0}(t) \equiv \bar{W}(t)$ is the Wronskian of the fundamental solution system $y_{10}(t), y_{20}(t), \ldots, y_{n-1,0}(t)$ of (13). With regard to (15) and (17), the expression (16) acquires the following form:

$$
\begin{aligned}
W(t, \varepsilon)= & (-1)^{1+n} \exp \left(\frac{1}{\varepsilon} \int_{0}^{t} \mu(x) d x\right) \\
& \times y_{n 0}(t)\left(W_{10}(t)+O(\varepsilon)\right) \\
& +\frac{(-1)^{2+n}}{\varepsilon} \exp \left(\frac{1}{\varepsilon} \int_{0}^{t} \mu(x) d x\right) \\
& \cdot y_{n 0}(t) \mu(t)\left(W_{20}(t)+O(\varepsilon)\right)+\cdots \\
& +(-1)^{2 n-1} \frac{1}{\varepsilon^{n-2}} \exp \left(\frac{1}{\varepsilon} \int_{0}^{t} \mu(x) d x\right) \\
& \cdot y_{n 0}(t) \mu^{n-2}(t) \cdot\left(W_{n-1,0}(t)+O(\varepsilon)\right) \\
& +(-1)^{2 n} \frac{1}{\varepsilon^{n-1}} \exp \left(\frac{1}{\varepsilon} \int_{0}^{t} \mu(x) d x\right) \\
& \cdot y_{n 0}(t) \mu^{n-1}(t)(\bar{W}(t)+O(\varepsilon)) .
\end{aligned}
$$


Hence, considering that the last summand is dominant, when $\varepsilon \rightarrow 0$ for the Wronskian determinant, it implies the asymptotic representation

$$
\begin{aligned}
W(t, \varepsilon)= & \frac{\bar{W}(t)}{\varepsilon^{n-1}} y_{n 0}(t) \mu^{n-1}(t) \cdot \exp \left(\frac{1}{\varepsilon} \int_{0}^{t} \mu(x) d x\right) \\
& \times(1+O(\varepsilon)) \neq 0, \quad 0 \leq t \leq 1,
\end{aligned}
$$

where $y_{n 0}(t)$ is given by (14).

\section{Cauchy Function and Boundary Functions}

Following previous work [17], let us introduce the Cauchy function.

Definition 2. Function $K(t, s, \varepsilon)$, defined at $0 \leq s \leq t \leq$ 1 , is called the Cauchy function of (10), if it satisfies the homogeneous equation (10) according to $t$ and within $t=s$ initial conditions:

$$
K^{(j)}(s, s, \varepsilon)=0, \quad j=\overline{0, n-2}, \quad K^{(n-1)}(s, s, \varepsilon)=1 .
$$

The following theorem is valid.

Theorem 3. Let conditions (a) and (b) be satisfied. Then, for sufficiently small $\varepsilon$, the Cauchy function $K(t, s, \varepsilon)$ with $0 \leq s \leq$ $t \leq 1$ exists, is unique, and is expressed as follows:

$$
K(t, s, \varepsilon)=\frac{W(t, s, \varepsilon)}{W(s, \varepsilon)},
$$

where $W(t, s, \varepsilon)$ is the nth order determinant obtained from the Wronskian $W(s, \varepsilon)$ by replacing the nth row of the fundamental solution system with $y_{1}(t, \varepsilon), y_{2}(t, \varepsilon), \ldots, y_{n}(t, \varepsilon)$ of $(10)$.

Lemma 4. If conditions (a) and (b) are satisfied, then, for sufficiently small $\varepsilon$, the Cauchy function $K(t, s, \varepsilon)$ with $0 \leq s \leq$ $t \leq 1$ can be represented in the following form:

$$
\begin{aligned}
& K_{t}^{(q)}(t, s, \varepsilon)= \frac{\varepsilon}{\mu(s) \bar{W}(s)} \cdot\left(-W_{n-1,0}^{(q)}(t, s)+O(\varepsilon)\right), \overline{0, n-3}, \\
& K_{t}^{(n-2)}(t, s, \varepsilon)=\varepsilon\left(-\frac{W_{n-1,0}^{(n-2)}(t, s)}{\mu(s) \bar{W}(s)}+\frac{y_{n 0}(t) \mu^{n-2}(t)}{y_{n 0}(s) \mu^{n-1}(s)}\right. \\
&\left.\quad \times \exp \left(\frac{1}{\varepsilon} \int_{s}^{t} \mu(x) d x\right)+O(\varepsilon)\right), \\
& K_{t}^{(n-1)}(t, s, \varepsilon)=-\varepsilon \frac{W_{n-1,0}^{(n-1)}(t, s)}{\mu(s) \bar{W}(s)} \\
&+\frac{y_{n 0}(t) \mu^{n-1}(t)}{y_{n 0}(s) \mu^{n-1}(s)} e^{(1 / \varepsilon) \int_{s}^{t} \mu(x) d x} \\
&+O\left(\varepsilon^{2}+\varepsilon \exp \left(\frac{1}{\varepsilon} \int_{s}^{t} \mu(x) d x\right)\right),
\end{aligned}
$$

where $W_{n-1,0}(t, s)$ is the determinant obtained from the Wronskian $\bar{W}(s)$ by substituting the $(n-1)$ th row with the $y_{i 0}(t), i=$ $\overline{1, n-1}$ row.

Proof. By expanding $W_{t}^{(q)}(t, s, \varepsilon), q=\overline{0, n-1}$ in entries of the $n$th column, we obtain

$$
\begin{aligned}
W_{t}^{(q)}(t, s, \varepsilon)= & \sum_{k=1}^{n-1}(-1)^{n+k} y_{n}^{(k-1)}(s, \varepsilon) \\
& \times \widetilde{W}_{k}^{(q)}(t, s, \varepsilon)+(-1)^{2 n} y_{n}^{(q)}(t, \varepsilon) \widetilde{W}_{n}(s, \varepsilon) .
\end{aligned}
$$

From (15), the minors $\widetilde{W}_{k}^{(q)}(t, s, \varepsilon), \widetilde{W}_{n}(s, \varepsilon)$ for sufficiently small $\varepsilon>0$ can be represented in the following form:

$$
\begin{gathered}
\widetilde{W}_{k}^{(q)}(t, s, \varepsilon)=W_{k 0}^{(q)}(t, s)+O(\varepsilon), \quad k=\overline{1, n-1} \\
\widetilde{W}_{n}(s, \varepsilon)=W_{n 0}(s)+O(\varepsilon)
\end{gathered}
$$

where $W_{n 0}(s)=\bar{W}(s)$ and $W_{k 0}^{(q)}(t, s)$ is the determinant obtained from the rectangular matrix $\left(y_{j 0}^{(i-1)}(s)\right)(i=$ $1, \ldots, n, j=1, \ldots, n-1)$ by deleting the $k$ th row and replacing the $(n-1)$ th row with the $\left(y_{10}^{(q)}(s), \ldots, y_{n-10}^{(q)}(s)\right)$ row.

Then, from (23) and (15), relation (24) acquires the following form:

$$
\begin{aligned}
W_{t}^{(q)}(t, s, \varepsilon)= & \sum_{k=1}^{n-1}(-1)^{n+k} \frac{1}{\varepsilon^{k-1}} \exp \left(\frac{1}{\varepsilon} \int_{0}^{s} \mu(x) d x\right) \\
& \quad \times y_{n 0}(s) \mu^{k-1}(s)\left(W_{k 0}^{(q)}(t, s)+O(\varepsilon)\right) \\
& +(-1)^{2 n} \frac{1}{\varepsilon^{q}} \exp \left(\frac{1}{\varepsilon} \int_{0}^{t} \mu(x) d x\right) \\
& \times y_{n 0}(t) \mu^{q}(t)(\bar{W}(s)+O(\varepsilon)) .
\end{aligned}
$$

This readily implies that

$$
\begin{aligned}
W_{t}^{(q)}(t, s, \varepsilon)= & \frac{1}{\varepsilon^{n-2}} y_{n 0}(s) \mu^{n-1}(s) \exp \left(\frac{1}{\varepsilon} \int_{0}^{s} \mu(x) d x\right) \\
\times & {\left[-\frac{W_{n-1,0}^{(q)}(t, s)}{\mu(s)}+\frac{\varepsilon^{n-2}}{\varepsilon^{q}} \frac{y_{n 0}(t) \mu^{q}(t)}{y_{n 0}(s) \mu^{n-1}(s)}\right.} \\
& \times \bar{W}(s) \exp \left(\frac{1}{\varepsilon} \int_{s}^{t} \mu(x) d x\right) \\
& \left.+O\left(\varepsilon+\varepsilon^{n-1-q} \exp \left(\frac{1}{\varepsilon} \int_{s}^{t} \mu(x) d x\right)\right)\right]
\end{aligned}
$$

From (26) with regard to (19) and (21), we obtain the desired estimates (22). 
Definition 5. Functions $\Phi_{k}(t, \varepsilon), k=\overline{1, n}$ are referred to as boundary functions of the boundary value problems (1) and (2), if they satisfy the homogeneous equation (10) and the boundary conditions

$$
L_{i} \Phi_{k}=\delta_{i j}, \quad i, j=1, \ldots, n .
$$

Consider the determinant

$$
J(\varepsilon)=\operatorname{det}\left(L_{k} y_{l}\right), \quad k, l=1, \ldots, n,
$$

where with (15), the entries $L_{i} y_{k}=\sum_{j=0}^{m_{i}} \alpha_{i j} y_{k}^{(j)}(0, \varepsilon)+$ $\sum_{j=0}^{r_{i}} \beta_{i j} y_{k}^{(j)}(1, \varepsilon)$ can be represented in the following form:

$$
\begin{array}{r}
L_{i} y_{k}=\sum_{j=0}^{m_{i}} \alpha_{i j} y_{k 0}^{(j)}(0)+\sum_{j=0}^{r_{i}} \beta_{i j} y_{k 0}^{(j)}(1)+O(\varepsilon), \\
i=\overline{1, n}, k=\overline{1, n-1} \\
L_{i} y_{n}=\sum_{j=0}^{m_{i}} \frac{\mu^{j}(0)}{\varepsilon^{j}} \alpha_{i j}(1+O(\varepsilon))+\sum_{j=0}^{r_{i}} \frac{\mu^{j}(1)}{\varepsilon^{j}} \beta_{i j} y_{n 0}(1) \\
\times \exp \left(\frac{1}{\varepsilon} \int_{0}^{1} \mu(x) d x\right)(1+O(\varepsilon))
\end{array}
$$

This, together with the estimate $\exp \left((1 / \varepsilon) \int_{0}^{1} \mu(x) d x\right)=o\left(\varepsilon^{N}\right)$ (where $N$ is an arbitrary positive integer), implies that

$$
\begin{array}{r}
L_{i} y_{k}=L_{i} y_{k 0}+O(\varepsilon), \quad L_{i} y_{n}=\frac{\mu^{m_{i}}(0)}{\varepsilon^{m_{i}}}\left(\alpha_{i m_{i}}+O(\varepsilon)\right), \\
i=\overline{1, n}, \quad k=\overline{1, n-1},
\end{array}
$$

where

$$
\begin{array}{r}
L_{i} y_{k 0}=\sum_{j=0}^{m_{i}} \alpha_{i j} y_{k 0}^{(j)}(0)+\sum_{j=0}^{r_{i}} \beta_{i j} y_{k 0}^{(j)}(1), \quad i=\overline{1, n} \\
k=\overline{1, n-1} .
\end{array}
$$

Now, let us expand $J(\varepsilon)$ in the entries of the last column:

$$
J(\varepsilon)=(-1)^{1+n} L_{1} y_{n} \cdot J_{1}(\varepsilon)+\cdots+(-1)^{2 n} L_{n} y_{n} \cdot J_{n}(\varepsilon) .
$$

Here, from (15), the minors $J_{i}(\varepsilon)$ can be expressed in the following form:

$$
J_{i}(\varepsilon)=\bar{J}_{0 i}+O(\varepsilon), \quad i=\overline{1, n},
$$

where $\bar{J}_{0 i}$ is the $(n-1)$ th-order determinant introduced in Section 1.

By substituting (30) and (33) into (32), we obtain

$$
\begin{aligned}
J(\varepsilon)= & (-1)^{1+n} \frac{\mu^{m_{1}}(0)}{\varepsilon^{m_{1}}}\left(\alpha_{1 m_{1}} \bar{J}_{01}+O(\varepsilon)\right)+\cdots \\
& +(-1)^{2 n} \frac{\mu^{m_{n}}(0)}{\varepsilon^{m_{n}}}\left(\alpha_{n m_{n}} \bar{J}_{0 n}+O(\varepsilon)\right) .
\end{aligned}
$$

This, together with condition (c), implies that the determinant (28) permits the asymptotic representation:

$$
J(\varepsilon)=(-1)^{1+n} \frac{1}{\varepsilon^{m_{1}}} \alpha_{1 m_{1}} \mu^{m_{1}}(0) \bar{J}_{01}(1+O(\varepsilon)) \neq 0 .
$$

The following theorem is valid.

Theorem 6. Let conditions (a)-(c) be satisfied. Then, for sufficiently small $\varepsilon>0$, the boundary functions $\Phi_{k}(t, \varepsilon), k=$ $\overline{1, n}$ exist on the interval $[0,1]$, are unique, and are given as follows:

$$
\Phi_{k}(t, \varepsilon)=\frac{J_{k}(t, \varepsilon)}{J(\varepsilon)}, \quad k=\overline{1, n}
$$

where $J_{k}(t, \varepsilon)$ is the determinant obtained from $J(\varepsilon)$ by substituting the kth row with the fundamental solution system $y_{i}(t, \varepsilon), i=\overline{1, n}$ of $(10)$.

Lemma 7. If conditions (a)-(c) are satisfied, then the boundary functions $\Phi_{k}(t, \varepsilon), k=\overline{1, n}$ permit the asymptotic representations:

$$
\begin{aligned}
\Phi_{1}^{(q)}(t, \varepsilon)=\frac{1}{\bar{J}_{01}}[- & -\varepsilon^{m_{1}-m_{2}} \frac{\alpha_{2 m_{2}} \mu^{m_{2}}(0)}{\alpha_{1 m_{1}} \mu^{m_{1}}(0)} \bar{J}_{12}^{(q)}(t) \\
& +\varepsilon^{m_{1}-q} \frac{y_{n 0}(t)}{\alpha_{1 m_{1}}} \frac{\mu^{q}(t)}{\mu^{m_{1}}(0)} \bar{J}_{01} \\
& \times \exp \left(\frac{1}{\varepsilon} \int_{0}^{t} \mu(x) d x\right) \\
& +O\left(\varepsilon^{1+m_{1}-m_{2}}+\varepsilon^{m_{1}+1-q}\right. \\
& \left.\left.\quad \times \exp \left(\frac{1}{\varepsilon} \int_{0}^{t} \mu(x) d x\right)\right)\right]
\end{aligned}
$$

$$
\begin{aligned}
& \Phi_{k}^{(q)}(t, \varepsilon)= \frac{\bar{J}_{k-1,1}^{(q)}(t)}{\bar{J}_{01}}+(-1)^{k-1} \varepsilon^{m_{1}-q} \frac{y_{n 0}(t) \mu^{q}(t)}{\alpha_{1 m_{1}} \mu^{m_{1}}(0)} \\
& \cdot \frac{\bar{J}_{0 k}}{\bar{J}_{01}} \exp \left(\frac{1}{\varepsilon} \int_{0}^{t} \mu(x) d x\right) \\
&+O\left(\varepsilon+\varepsilon^{m_{1}+1-q} \exp \left(\frac{1}{\varepsilon} \int_{0}^{t} \mu(x) d x\right)\right), \\
& k=2, \ldots, n, q=\overline{0, n-1},
\end{aligned}
$$

on the closed interval $[0,1]$ as $\varepsilon \rightarrow 0$, where $\bar{J}_{01}$ and $\bar{J}_{0 k}$ are the determinants introduced in Section $1, \bar{J}_{12}^{(q)}(t)=\bar{J}_{21}^{(q)}(t)$, and $\bar{J}_{k-1,1}^{(q)}(t)$ is the $(n-1)$ th-order determinant obtained from $\bar{J}_{01}$ by replacing the $(k-1)$ th row with the $y_{10}^{(q)}(t), \ldots, y_{n-10}^{(q)}(t)$ row. 
Proof. From (36), we define the derivative $J_{1}^{(q)}(t, \varepsilon)$ of the function $J_{1}(t, \varepsilon)$. Let us expand $J_{1}^{(q)}(t, \varepsilon)$ in the entries of the $n$th column:

$$
\begin{aligned}
J_{1}^{(q)}(t, \varepsilon)= & (-1)^{1+n} y_{n}^{(q)}(t, \varepsilon) J_{01 n}(\varepsilon) \\
& +(-1)^{2+n} L_{2} y_{n} J_{12 n}(t, \varepsilon)+\cdots \\
& +(-1)^{2 n} L_{n} y_{n} J_{1 n n}(t, \varepsilon) .
\end{aligned}
$$

Here in the minors $J_{011}(\varepsilon), J_{1 j n}(t, \varepsilon),(j=\overline{1, n})$, the first subscript represents the number of the row containing the system of functions $y_{1}^{(q)}(t, \varepsilon), \ldots, y_{n-1}^{(q)}(t, \varepsilon)$. From (30), the determinants $J_{011}(\varepsilon), J_{1 j n}(t, \varepsilon),(j=\overline{1, n})$ can be presented in the following form:

$$
\begin{array}{r}
J_{011}(\varepsilon)=\bar{J}_{01}+O(\varepsilon), \quad J_{1 j n}(t, \varepsilon)=\bar{J}_{1 j}^{(q)}(t)+O(\varepsilon), \\
j=\overline{2, n},
\end{array}
$$

where the determinants $\bar{J}_{1 j}^{(q)}(t)$ are obtained from $\bar{J}_{0 j}$ by substituting the first row with the $y_{10}^{(q)}(t) \cdots y_{n-1,0}^{(q)}(t)$ row.

With regard to (15), (30), and (40), the expression $J_{1}^{(q)}(t, \varepsilon)$ acquires the following form:

$$
\begin{aligned}
J_{1}^{(q)}(t, \varepsilon)= & (-1)^{1+n} \frac{1}{\varepsilon^{q}} \exp \left(\frac{1}{\varepsilon} \int_{0}^{t} \mu(x) d x\right) \\
& \times \mu^{q}(t) y_{n 0}(t)\left(\bar{J}_{01}+O(\varepsilon)\right)+(-1)^{2+n} \\
& \times \frac{\mu^{m_{2}}(0)}{\varepsilon^{m_{2}}}\left(\alpha_{2 m_{2}} \bar{J}_{12}^{(q)}(t)+O(\varepsilon)\right)+\cdots \\
& +(-1)^{2 n} \frac{\mu^{m_{n}}(0)}{\varepsilon^{m_{n}}}\left(\alpha_{n m_{n}} \bar{J}_{1 n}^{(q)}(t)+O(\varepsilon)\right), \\
q & =\overline{0, n-1} .
\end{aligned}
$$

This, together with the condition (c), gives the following representation:

$$
\begin{aligned}
J_{1}^{(q)}(t, \varepsilon)= & \frac{(-1)^{n+1}}{\varepsilon^{m_{2}}} \\
\times & \times\left[-\alpha_{2 m_{2}} \mu^{m_{2}}(0) \bar{J}_{12}^{(q)}(t)\right. \\
& \quad+\varepsilon^{m_{2}-q} \mu^{q}(t) y_{n 0}(t) \bar{J}_{01} \exp \left(\frac{1}{\varepsilon} \int_{0}^{t} \mu(x) d x\right) \\
& \left.+O\left(\varepsilon+\varepsilon^{m_{2}+1-q} \exp \left(\frac{1}{\varepsilon} \int_{0}^{t} \mu(x) d x\right)\right)\right], \\
& \quad q=\overline{0, n-1} .
\end{aligned}
$$

Taking into account (35), (42), and (36), we obtain relation (37) for $\Phi_{1}^{(q)}(t, \varepsilon)$.
Now, we expand $J_{k}^{(q)}(t, \varepsilon)$ in the entries of the $n$th column:

$$
\begin{aligned}
J_{k}^{(q)}(t, \varepsilon)= & (-1)^{1+n} L_{1} y_{n} J_{k-1,1}(t, \varepsilon)+\cdots \\
& +(-1)^{k-1+n} L_{k-1} y_{n} J_{k-1, k-1}(t, \varepsilon) \\
& +(-1)^{k+n} y_{n}^{(q)}(t, \varepsilon) J_{0 k}(\varepsilon) \\
& +(-1)^{k+1+n} L_{k+1} y_{n} J_{k, k+1}(t, \varepsilon) \\
& +\cdots+(-1)^{2 n} L_{n} y_{n} J_{k n}(t, \varepsilon) .
\end{aligned}
$$

Here the first subscript in the determinants $J_{0 k}, J_{k-1, i}^{(q)}(i=$ $\overline{1, k-1)}, J_{k i}^{(q)}(i=\overline{k+1, n})$ indicates the row containing the system of the functions $y_{1}^{(q)}(t, \varepsilon) \cdots y_{n-1}^{(q)}(t, \varepsilon)$, and the second subscript shows that they are the minors of the entry of the determinant $J_{k}^{(q)}(t, \varepsilon)$ at the intersection of the $i$ th row and $n$th column. From (30), the minors $J_{0 k}, J_{k-1, i}^{(q)}(i=$ $\overline{1, k-1}), J_{k i}^{(q)}(i=\overline{k+1, n})$ can be presented in the following form:

$$
\begin{array}{r}
J_{0 k}(\varepsilon)=\bar{J}_{0 k}+O(\varepsilon), \quad J_{k-1, i}^{(q)}(t, \varepsilon)=\bar{J}_{k-1, i}^{(q)}(t)+O(\varepsilon), \\
i=\overline{1, k-1,} \\
J_{k i}^{(q)}(t, \varepsilon)=\bar{J}_{k i}^{(q)}(t)+O(\varepsilon), \quad i=\overline{k+1, n,}
\end{array}
$$

where $\bar{J}_{0 k}$ is as defined in Section $1, \bar{J}_{k-1, i}^{(q)}(t)(i=\overline{1, k-1})$ is obtained from $\bar{J}_{0 i}(i=1, \ldots, k-1)$ by replacing the $(k-1)$ th row with the $y_{10}^{(q)}(t) \cdots y_{n-1,0}^{(q)}(t)$ row, and $\bar{J}_{k i}^{(q)}(t)(i=\overline{k+1, n})$ is obtained from $\bar{J}_{0 i}(i=k+1, \ldots, n)$ by replacing the $k$ th with the $y_{10}^{(q)}(t) \cdots y_{n-1,0}^{(q)}(t)$ row.

In view of (30) and (44) out of (43), the expression takes the following form:

$$
\begin{aligned}
J_{k}^{(q)}(t, \varepsilon)= & (-1)^{1+n} \frac{\mu^{m_{1}}(0)}{\varepsilon^{m_{1}}}\left(\alpha_{1 m_{1}} \bar{J}_{k-1,1}^{(q)}(t)+O(\varepsilon)\right)+\cdots \\
& +(-1)^{k-1+n} \frac{\mu^{m_{k-1}}(0)}{\varepsilon^{m_{k-1}}} \\
& \times\left(\alpha_{k-1, m_{k-1}} \bar{J}_{k-1, k-1}^{(q)}(t)+O(\varepsilon)\right) \\
& +(-1)^{k+n} \frac{y_{n 0}(t)}{\varepsilon^{q}} \mu^{q}(t) \exp \left(\frac{1}{\varepsilon} \int_{0}^{t} \mu(x) d x\right) \\
& \times\left(\bar{J}_{0 k}+O(\varepsilon)\right)+(-1)^{k+1+n} \\
& \times \frac{\mu^{m_{k+1}}(0)}{\varepsilon^{m_{k+1}}}\left(\alpha_{k+1, m_{k+1}} \bar{J}_{k, k+1}^{(q)}(t)+O(\varepsilon)\right)+\cdots \\
& +(-1)^{2 n} \frac{\mu^{m_{n}}(0)}{\varepsilon^{m_{n}}} \\
& \times\left(\alpha_{n m_{n}} \bar{J}_{k n}^{(q)}(t)+O(\varepsilon)\right), \quad q=\overline{0, n-1} .
\end{aligned}
$$


Here the expression $(-1)^{1+n}\left(\mu^{m_{1}}(0) / \varepsilon^{m_{1}}\right) \alpha_{1 m_{1}} \bar{J}_{k-1,1}^{(q)}(t)$ is dominant for small $\varepsilon$. Then, considering condition (c), we get the following asymptotic representation for $J_{k}^{(q)}(t, \varepsilon)$ :

$$
\begin{aligned}
J_{k}^{(q)}(t, \varepsilon)= & \frac{(-1)^{n+1}}{\varepsilon^{m_{1}}} \\
& \times\left[\alpha_{1 m_{1}} \mu^{m_{1}}(0) \bar{J}_{k-1,1}^{(q)}(t)\right. \\
& \quad+(-1)^{k-1} \varepsilon^{m_{1}-q} \mu^{q}(t) y_{n 0}(t) \bar{J}_{0 k} \\
& \quad \times \exp \left(\frac{1}{\varepsilon} \int_{0}^{t} \mu(x) d x\right) \\
& \left.+O\left(\varepsilon+\varepsilon^{m_{1}+1-q} \exp \left(\frac{1}{\varepsilon} \int_{0}^{t} \mu(x) d x\right)\right)\right], \\
& \quad k=\overline{2, n}, q=\overline{0, n-1 .}
\end{aligned}
$$

Substituting (35) and (46) into formula (36), we obtain the asymptotic formula (38) for the boundary functions $\Phi_{k}^{(q)}(t, \varepsilon)$. The proof of the lemma is complete.

\section{Analytic Representation and Estimates of the Solution}

Consider the singularly perturbed boundary value problems (1) and (2). The unique solution of the problems (1) and (2) is found in the following form:

$$
\begin{aligned}
y(t, \lambda, \varepsilon)= & c_{1} \Phi_{1}(t, \varepsilon)+\cdots+c_{n} \Phi_{n}(t, \varepsilon) \\
& +\frac{\lambda}{\varepsilon} \int_{0}^{t} K(t, s, \varepsilon) h(s) d s
\end{aligned}
$$

where $\Phi_{k}(t, \varepsilon), k=\overline{1, n}$ are the boundary functions, $K(t, s, \varepsilon)$ is the Cauchy function, $c_{1}, c_{2}, \ldots, c_{n}$ are unknown consonants, and $\lambda$ is the required parameter. By a straightforward verification, one can show that the function $y(t, \lambda, \varepsilon)$, defined by formula (47), is identical to (1). For the determination of $c_{1}, c_{2}, \ldots, c_{n}, \lambda$, we substitute (47) into the boundary conditions (b). Then, with regard to the boundary (27), we obtain the following system of linear equations with respect to $c_{1}, c_{2}, \ldots, c_{n}, \lambda$ as follows:

$$
\begin{gathered}
c_{i}=a_{i}-\sum_{j=0}^{r_{i}} \beta_{i j} \frac{\lambda}{\varepsilon} \int_{0}^{1} K_{t}^{(j)}(1, s, \varepsilon) h(s) d s, \quad i=1, \ldots, n \\
\lambda D=a_{n+1}-\sum_{i=1}^{n} a_{i} L_{n+1} \Phi_{i}(t, \varepsilon)
\end{gathered}
$$

where

$$
\begin{gathered}
D=D_{n+1}-\sum_{i=1}^{n} D_{i} L_{n+1} \Phi_{i}(t, \varepsilon), \\
D_{i}=\sigma_{i}+\frac{1}{\varepsilon} \sum_{j=0}^{r_{i}} \beta_{i j} \int_{0}^{1} h(s) K_{t}^{(j)}(1, s, \varepsilon) d s, \quad i=\overline{1, n+1} .
\end{gathered}
$$

We study the coefficient in (49) for $\lambda$ and the right-hand side of this equation as a function of $\varepsilon$. Then, with regard to (27), (35), (37), and (38), we obtain

$$
\begin{gathered}
D=\bar{D}+O(\varepsilon), \\
a_{n+1}-\sum_{i=1}^{n} a_{i} L_{n+1} \Phi_{i}(t, \varepsilon) \\
=a_{n+1}-\sum_{i=2}^{n} \frac{a_{i} L_{n+1} \bar{J}_{i-1}(t)}{\bar{J}_{01}}+O(\varepsilon) .
\end{gathered}
$$

From condition (d), $\bar{D} \neq 0$. Therefore, for sufficiently small $\varepsilon$,

$$
D \neq 0 .
$$

Consequently, for sufficiently small $\varepsilon>0,(49)$ is uniquely solvable and can be represented in the following form:

$$
\lambda=\tilde{\lambda}(\varepsilon)=\frac{1}{D}\left(a_{n+1}-\sum_{i=1}^{n} a_{i} L_{n+1} \Phi_{i}(t, \varepsilon)\right),
$$

and from (51) and (52), the fair estimate for it is

$$
\tilde{\lambda}(\varepsilon)=\lambda_{0}+O(\varepsilon)
$$

where

$$
\lambda_{0}=\frac{1}{\bar{D}}\left(a_{n+1}-\sum_{i=1}^{n} \frac{a_{i} L_{n+1} \bar{J}_{i-1}(t)}{\bar{J}_{01}}\right) .
$$

Now, substituting (54) into (48), we explicitly determine

$$
c_{i}=a_{i}-\sum_{j=0}^{r_{i}} \beta_{i j} \frac{\tilde{\lambda}(\varepsilon)}{\varepsilon} \int_{0}^{1} K_{t}^{(j)}(1, s, \varepsilon) h(s) d s, \quad i=1, \ldots, n .
$$

Using (54), (57), and (50) out of (47), we obtain the representation, and for the component $y$ of the problem solutions (1) and (2),

$$
\begin{aligned}
& y(t, \tilde{\lambda}(\varepsilon), \varepsilon) \\
& =\sum_{i=1}^{n} a_{i} \Phi_{i}(t, \varepsilon)-\tilde{\lambda}(\varepsilon) \\
& \quad \times \sum_{i=1}^{n}\left(\sigma_{i}+\sum_{j=0}^{r_{i}} \frac{\beta_{i j}}{\varepsilon} \int_{0}^{1} K_{t}^{(j)}(1, s, \varepsilon) h(s) d s\right) \\
& \quad \times \Phi_{i}(t \cdot \varepsilon)+\frac{\tilde{\lambda}(\varepsilon)}{\varepsilon} \int_{0}^{t} K(t, s, \varepsilon) h(s) d s .
\end{aligned}
$$

Thus, the following theorem is valid. 
Theorem 8. Let conditions (a)-(d) be satisfied. Then, for sufficiently small $\varepsilon>0$ in a sufficiently small neighborhood of the point $\lambda=\lambda_{0}$, a unique value of $\lambda=\widetilde{\lambda}(\varepsilon)$ can be determined such that the pair $(y(t, \widetilde{\lambda}(\varepsilon), \varepsilon), \tilde{\lambda}(\varepsilon))$ is the unique solution for the boundary value problems (1) and (2) on the interval $[0,1]$, where $y(t, \widetilde{\lambda}(\varepsilon), \varepsilon)$ is given by formula $(58)$ and $\widetilde{\lambda}(\varepsilon)$ is given by formula (54).

Theorem 9. Let conditions (a)-(e) be satisfied. Then, for sufficiently small $\varepsilon$, the solution $y(t, \varepsilon)$ of the boundary value problems (1) and (2) and its derivatives on the interval $0 \leq t \leq$ 1 can be estimated as follows:

$$
\begin{aligned}
& \left|y^{(q)}(t, \tilde{\lambda}(\varepsilon), \varepsilon)\right| \\
& \leq C\left[\left|a_{1}-\tilde{\lambda} \sigma_{1}\right| \cdot\left(\varepsilon^{m_{1}-m_{2}}+\varepsilon^{m_{1}-q} \exp \left(-\frac{\gamma t}{\varepsilon}\right)\right)\right. \\
& \quad+\sum_{i=2}^{n}\left|a_{i}-\tilde{\lambda} \sigma_{i}\right|+|\widetilde{\lambda}| \max _{0 \leq t \leq 1}|h(t)|+\varepsilon^{m_{1}-q} \\
& \left.\quad \times \exp \left(-\frac{\gamma t}{\varepsilon}\right)\left(\sum_{i=2}^{n}\left|a_{i}-\tilde{\lambda} \sigma_{i}\right|+|\widetilde{\lambda}| \max _{0 \leq t \leq 1}|h(t)|\right)\right], \\
& q=\overline{0, n-1},
\end{aligned}
$$

where $C>0$ and $\gamma>0$ are the constants independent of $\varepsilon$.

Proof. Formulae (22), (37), and (38) together with (5) imply the following estimates:

$$
\begin{gathered}
\left|K_{t}^{(q)}(t, s, \varepsilon)\right| \leq C \varepsilon, \quad q=\overline{0, n-2}, \quad 0 \leq s \leq t \leq 1, \\
\left|K_{t}^{(n-1)}(t, s, \varepsilon)\right| \leq C\left(\varepsilon+\exp \left(-\frac{\gamma(t-s)}{\varepsilon}\right)\right), \\
0 \leq s \leq t \leq 1, \\
\left|\Phi_{i}^{(q)}(t, \varepsilon)\right| \leq C \cdot\left(1+\varepsilon^{m_{1}-q} \cdot \exp \left(-\frac{\gamma t}{\varepsilon}\right)\right), \quad i=\overline{2, n}, \\
q=\overline{0, n-1}, 0 \leq t \leq 1, \\
\left|\Phi_{1}^{(q)}(t, \varepsilon)\right| \leq C\left(\varepsilon^{m_{1}-m_{2}}+\varepsilon^{m_{1}-q} \cdot \exp \left(-\frac{\gamma t}{\varepsilon}\right)\right), \\
q=\overline{0, n-1}, 0 \leq t \leq 1 .
\end{gathered}
$$

By estimating solution (58) and taking into account (60), we obtain (59), which completes the proof of the theorem.

Now, let us formulate the boundary conditions for the following unperturbed (degenerate) equation:

$$
L_{0} \bar{y}=A_{1}(t) \bar{y}^{(n-1)}+\cdots+A_{n}(t) \bar{y}(t)=h(t),
$$

obtained from (1) within $\varepsilon=0$. On the basis of Theorem 9, we must conclude that in (58), the coefficient of $a_{1}$ approaches zero when $\varepsilon \rightarrow 0$ and the coefficients of $a_{i}, i=\overline{2, n}$ have the order $O(1)$. Therefore, the boundary conditions for the solution of the unperturbed (61) are defined with help of the boundary conditions (2), containing $a_{2}, \ldots, a_{n+1}$ :

$$
L_{2} \bar{y}+\sigma_{2} \lambda=a_{2}, \ldots, L_{n+1} \bar{y}+\sigma_{n+1} \lambda=a_{n+1} .
$$

Next, we show that (61) and boundary conditions (62) actually define the degenerate problem.

By analogy with (17) and (28) for the problems (51) and (52), we introduce the initial function and boundary functions:

$$
\bar{K}(t, s)=\frac{\bar{W}(t, s)}{\bar{W}(s)}, \quad \bar{\Phi}_{k-1}(t)=\frac{\bar{J}_{k-1}(t)}{\bar{J}_{01}}, \quad k=\overline{2, n}
$$

where $\bar{J}_{01}$ is the determinant $(6), \bar{J}_{k-1}(t)=\bar{J}_{k-1,1}^{(0)}(t)$ is the determinant from Lemma $7, \bar{W}(t, s) \equiv W_{n-1,0}(t, s)$ is the determinant from Lemma 4 , and $\bar{W}(s) \equiv W_{n 0}(s)$ is the Wronskian determinant of the fundamental solution system $y_{i 0}(s), i=\overline{1, n-1}$ of homogeneous degenerate equation (13), which is obtained from $\bar{W}(s)$ by replacing the $(n-1)$ th row with the $y_{i 0}(t), i=\overline{1, n-1}$ row.

Apparently, $\bar{K}(t, s)$ is the Cauchy function satisfying the homogeneous equation $L_{0} \bar{K}(t, s)=0$ with respect to $t$ and initial conditions $\bar{K}_{t}^{(j)}(s, s)=0, j=\overline{0, n-3}, \bar{K}_{t}^{(n-2)}(s, s)=$ 1 , and $\bar{\Phi}_{k}(t), k=\overline{1, n-1}$ as the boundary functions of the boundary value problems (61) and (62):

$$
\begin{aligned}
L_{0} \bar{\Phi}_{k}(t)=0, & L_{i} \bar{\Phi}_{k}=1 \quad \text { within } i=k+1, \\
L_{i} \bar{\Phi}_{k} & =0 \quad \text { within } i \neq k+1, i=\overline{2, n} .
\end{aligned}
$$

Theorem 10. Let conditions (a)-(d) be satisfied. Then, the pair $\left(\bar{y}\left(t, \lambda_{0}\right), \lambda_{0}\right)$ is the unique solution of the nonhomogeneous boundary-value problems (61) and (62) on the interval $[0,1]$, where $\lambda_{0}$ is expressed by formula (56) and $\bar{y}\left(t, \lambda_{0}\right)$ is

$$
\begin{aligned}
\bar{y}\left(t, \lambda_{0}\right)= & \sum_{i=2}^{n}\left(a_{i}-\lambda_{0} \sigma_{i}\right) \bar{\Phi}_{i-1}(t) \\
& -\lambda_{0} \sum_{i=2}^{n} \bar{\Phi}_{i-1}(t) \sum_{j=0}^{r_{i}} \beta_{i j} \int_{0}^{1} \frac{\bar{K}_{t}^{(j)}(1, s)}{A_{1}(s)} h(s) d s \\
& +\lambda_{0} \int_{0}^{t} \frac{\bar{K}(t, s)}{A_{1}(s)} h(s) d s .
\end{aligned}
$$

Proof. We seek the solution $(\bar{y}(t, \lambda), \lambda)$ of the boundary value problems (61) and (62) in the following form:

$$
\begin{aligned}
\bar{y}(t, \lambda)= & c_{1} \bar{\Phi}_{1}(t)+\cdots+c_{n-1} \bar{\Phi}_{n-1}(t) \\
& +\lambda \int_{0}^{t} \frac{\bar{K}(t, s)}{A_{1}(s)} h(s) d s,
\end{aligned}
$$

where $\bar{\Phi}_{k}(t), k=\overline{1, n-1}$ are boundary functions, $\bar{K}(t, s)$ is the Cauchy function, $c_{1}, c_{2}, \ldots, c_{n-1}$ are unknown constants, 
and $\lambda$ is the required parameter. It is easy to verify that the function $\bar{y}(t, \lambda)$ identically satisfies (20). For the determination of $c_{1}, c_{2}, \ldots, c_{n-1}, \lambda$, let us substitute (51) into the boundary conditions (49). Then, with regard to the boundary conditions (26), we obtain the following system of linear equations with respect to $c_{1}, c_{2}, \ldots, c_{n-1}, \lambda$ :

$$
\begin{aligned}
& c_{1} L_{i} \bar{\Phi}_{1}(t)+\cdots+c_{n-1} L_{i} \bar{\Phi}_{n-1}(t) \\
& +\lambda\left(\sigma_{i}+\sum_{j=0}^{r_{i}} \beta_{i j} \int_{0}^{1} \frac{\bar{K}_{t}^{(j)}(1, s)}{A_{1}(s)} h(s) d s\right)=a_{i}, \\
& c_{1} L_{n+1} \bar{\Phi}_{1}(t)+\cdots+c_{n-1} L_{n+1} \bar{\Phi}_{n-1}(t) \\
& +\lambda\left(\sigma_{n+1}+\sum_{j=0}^{r_{n+1}} \beta_{n+1 j} \int_{0}^{1} \frac{\bar{K}_{t}^{(j)}(1, s)}{A_{1}(s)} h(s) d s\right)=a_{n+1} .
\end{aligned}
$$

Hence, with regard to (64), we have

$$
\begin{gathered}
c_{i-1}=a_{i}-\lambda\left(\sigma_{i}+\sum_{j=0}^{r_{i}} \beta_{i j} \int_{0}^{1} \frac{\bar{K}_{t}^{(j)}(1, s)}{A_{1}(s)} h(s) d s\right), \quad i=\overline{2, n} \\
\sum_{i=2}^{n} a_{i} L_{n+1} \bar{\Phi}_{i-1}(t) \\
+\lambda\left(\sigma_{n+1}+\sum_{j=0}^{r_{n+1}} \beta_{n+1 j} \int_{0}^{1} \frac{\bar{K}_{t}^{(j)}(1, s)}{A_{1}(s)} h(s) d s\right) \\
-\lambda\left(\sum_{i=2}^{n}\left(\sigma_{i}+\sum_{j=0}^{r_{i}} \beta_{i j} \int_{0}^{1} \frac{\bar{K}_{t}^{(j)}(1, s)}{A_{1}(s)} h(s) d s\right)\right. \\
\left.\times L_{n+1} \bar{\Phi}_{i-1}(t)\right)=a_{n+1} \cdot
\end{gathered}
$$

From (53), (58), and condition (d), we determine

$$
\lambda=\lambda_{0}=\frac{1}{\bar{D}}\left(a_{n+1}-\sum_{i=1}^{n} a_{i} L_{n+1} \bar{\Phi}_{i-1}(t)\right),
$$

where $\bar{D}=\bar{D}_{n+1}-\sum_{i=2}^{n} \bar{D}_{i} L_{n+1} \bar{\Phi}_{i-1} \neq 0, \bar{D}_{i}=\sigma_{i}+$ $\sum_{j=0}^{r_{i}} \beta_{i j} \int_{0}^{1}\left(h(s) / A_{1}(s)\right) \bar{K}_{t}^{(j)}(1, s) d s(i=\overline{1, n+1})$, and the values of $\lambda_{0}$ are given in Section 2.

Substituting (70) into (68), we definitely find

$$
\begin{array}{r}
c_{i-1}=a_{i}-\lambda_{0}\left(\sigma_{i}+\sum_{j=0}^{r_{i}} \beta_{i j} \int_{0}^{1} \frac{\bar{K}_{t}^{(j)}(1, s)}{A_{1}(s)} h(s) d s\right), \\
i=\overline{2, n} .
\end{array}
$$

Now, using (70) and (71) out of (66), we obtain the presentation (65). The proof of Theorem 10 is complete.
Theorem 11. Let conditions (a)-(e) be satisfied. Then, the estimates

$$
\begin{array}{r}
\left|y^{(q)}(t, \tilde{\lambda}, \varepsilon)-\bar{y}^{(q)}\left(t, \lambda_{0}\right)\right| \leq C\left(\varepsilon+\varepsilon^{m_{1}-q} \exp \left(-\frac{\gamma t}{\varepsilon}\right)\right), \\
t \in[0,1], q=\overline{0, n-1}
\end{array}
$$

are valid for sufficiently small $\varepsilon>0$, where $y(t, \tilde{\lambda}, \varepsilon)$ is the solution to the problems (1) and (2), and $\bar{y}\left(t, \lambda_{0}\right)$ is the solution of the problems (61) and (62).

Proof. Let $u(t, \varepsilon)=y(t, \tilde{\lambda}(\varepsilon), \varepsilon)-\bar{y}\left(t, \lambda_{0}\right)$, where $y(t, \tilde{\lambda}(\varepsilon), \varepsilon)$ is the solution of the problems (1) and (2) and $\bar{y}\left(t, \lambda_{0}\right)$ is the solution of the problems (61) and (62). We substitute the variable $y(t, \tilde{\lambda}(\varepsilon), \varepsilon)=u(t, \varepsilon)+\bar{y}\left(t, \lambda_{0}\right)$, which represents the problems (1) and (2) together with (61) and (62) in the following form:

$$
L_{\varepsilon} u=-\varepsilon \cdot \bar{y}^{(n)}(t)
$$

$$
\begin{gathered}
L_{i} u=\sum_{j=0}^{m_{i}} \alpha_{i j} u^{(j)}(0, \varepsilon)+\sum_{j=0}^{r_{i}} \beta_{i j} u^{(j)}(1, \varepsilon)=O(\varepsilon), \\
i=2, \ldots, n+1, \\
L_{1} u=\sum_{j=0}^{m_{1}} \alpha_{1 j} u^{(j)}(0, \varepsilon)+\sum_{j=0}^{r_{1}} \beta_{1 j} u^{(j)}(1, \varepsilon) \\
=a_{1}-\sigma_{1} \tilde{\lambda}(\varepsilon)-L_{1} \bar{y} .
\end{gathered}
$$

By applying Theorem 9 to this problem, we obtain

$$
\begin{array}{r}
\left|u^{(q)}(t, \varepsilon)\right| \leq C\left(\varepsilon+\varepsilon^{m_{1}-q} \exp \left(-\frac{\gamma t}{\varepsilon}\right)\right), \\
t \in[0,1], q=\overline{0, n-1 .}
\end{array}
$$

Now, we obtain the desired estimate (72). The proof of the theorem is complete.

Thus, from Theorem 11, it follows that the solution $y(t, \tilde{\lambda}, \varepsilon), \tilde{\lambda}$ of the boundary value problems (1) and (2) approaches the solution $\bar{y}\left(t, \lambda_{0}\right), \lambda_{0}$ of the degenerate problems (61) and (62) as the small parameter $\varepsilon$ approaches zero:

$$
\begin{gathered}
\lim _{\varepsilon \rightarrow 0} \tilde{\lambda}(\varepsilon)=\lambda_{0}, \\
\lim _{\varepsilon \rightarrow 0} y^{(q)}(t, \tilde{\lambda}, \varepsilon)=\bar{y}^{(q)}\left(t, \lambda_{0}\right), \quad 0 \leq t \leq 1, q=\overline{0, m_{1}-1}, \\
\lim _{\varepsilon \rightarrow 0} y^{(q)}(t, \tilde{\lambda}, \varepsilon)=\bar{y}^{(q)}\left(t, \lambda_{0}\right), \quad 0<t \leq 1, \\
q=m_{1}, m_{1}+1, \ldots, n-1 .
\end{gathered}
$$

Therefore, it follows that the problems (61) and (62) are degenerate problems.

Apparently, passages to the limit (75) are not even in the right neighborhood of the point $t=0$. If we specify the 
solution tendency of the original problems (1) and (2) to the solution of degenerate problems (61) and (62), we learn the nature of the growth of derivatives of the (1) and (2) problem solutions in the neighborhood of the point $t=0$ for $\varepsilon \rightarrow 0$. For this purpose, we use the representations of (22), (37), and (38) and formulae (58) and (65). Then, for the solution $y(t, \varepsilon)$ and its derivatives, $y^{(q)}(t, \varepsilon), q=0,1, \ldots, n-1$ of the problems (1) and (2) on the interval $0 \leq t \leq 1$, we obtain the following asymptotic representation:

$$
\begin{aligned}
y^{(q)}(t, \varepsilon)= & \sum_{i=2}^{n}\left(a_{i}-\lambda_{0} \sigma_{i}\right) \bar{\Phi}_{i-1}^{(q)}(t) \\
& \quad-\lambda_{0} \sum_{i=2}^{n} \bar{\Phi}_{i-1}^{(q)}(t) \sum_{j=0}^{r_{i}} \beta_{i j} \int_{0}^{1} \frac{\bar{K}_{t}^{(j)}(1, s)}{A_{1}(s)} h(s) d s \\
& +\lambda_{0} \int_{0}^{t} \frac{\bar{K}_{t}^{(q)}(t, s)}{A_{1}(s)} h(s) d s+\varepsilon^{m_{1}-q} \\
& \cdot \mathrm{e}^{(1 / \varepsilon) \int_{0}^{t} \mu(x) d x} \cdot \frac{y_{n 0}(t)}{\alpha_{1 m_{1}}} \cdot \frac{\mu^{q}(t)}{\mu^{m_{1}}(0)} \cdot \Delta\left(\lambda_{0}\right) \\
& +\varepsilon^{n-1-q} \cdot \lambda_{0}\left(-\frac{h(t) \cdot \mu^{q}(t)}{\mu^{n}(t)}+h(0)\right. \\
& +O\left(\varepsilon+\varepsilon^{m_{1}+1-q} \mathrm{e}^{(1 / \varepsilon) \int_{0}^{t} \mu(x) d x}\right) \cdot
\end{aligned}
$$

Here

$$
\Delta\left(\lambda_{0}\right)=\frac{\Delta_{0}\left(\lambda_{0}\right)}{\alpha_{1 m_{1}} \bar{J}_{01}} \neq 0,
$$

where $\Delta_{0}\left(\lambda_{0}\right)$ is the expression (9) from condition (d) as

$$
\begin{aligned}
& \Delta_{0}\left(\lambda_{0}\right) \\
& =\sum_{i=1}^{n}(-1)^{1+i} \bar{J}_{0 i} \\
& \quad \times\left(a_{i}-\lambda_{0} \sigma_{i}-\lambda_{0} \sum_{j=0}^{r_{i}} \beta_{i j} \int_{0}^{1} \frac{h(s)}{A_{1}(s)} \bar{K}_{t}^{(j)}(1, s) d s\right) .
\end{aligned}
$$

It is easy to say that the representation (78) is expressed in the following form:

$$
\Delta\left(\lambda_{0}\right)=\frac{\Delta_{0}\left(\lambda_{0}\right)}{\alpha_{1 m_{1}} \bar{J}_{01}}=\frac{1}{\alpha_{1 m_{1}}}\left(a_{1}-\lambda_{0} \sigma_{1}-L_{1} \bar{y}_{0}\right) \neq 0 .
$$

Now, from (79) and (76), it follows that the derivatives $y^{(m-j)}(t, \varepsilon), j=1, \ldots, n-m_{1}-1$ at point $t=0$ have poles for $\varepsilon$ :

$$
\begin{array}{r}
y^{\left(m_{1}+j\right)}(0, \varepsilon)=\frac{\mu^{j}(0)}{\varepsilon^{j}}\left(\Delta\left(\lambda_{0}\right)+O(\varepsilon)\right), \\
j=1, \ldots, n-1-m_{1},
\end{array}
$$

and $y^{\left(m_{1}\right)}(t, \varepsilon)$ at the point $t=0$ has the phenomenon of the $m_{1}$ th-order initial jump. Moreover, the magnitude of the jump is defined as

$$
\begin{aligned}
& \lim _{\varepsilon \rightarrow 0} y^{\left(m_{1}\right)}(0, \tilde{\lambda}, \varepsilon)-\bar{y}^{\left(m_{1}\right)}\left(0, \lambda_{0}\right) \\
& =\Delta\left(\lambda_{0}\right)=\frac{1}{\alpha_{m_{1}}}\left(a_{1}-\bar{\lambda}_{0} \sigma_{1}-L_{1} \bar{y}\right) .
\end{aligned}
$$

Thus, for the parameters $m_{1}=$ fix $\in\{0,1, \ldots, n-1\}$, a class exists for the restoration problem with an initial jump from the position of a singularly perturbed problem.

\section{Example, Remarks, and Conclusions}

(1) Let us consider the example of the boundary value problem exemplifying the problems (1) and (2) as

$$
\begin{gathered}
\varepsilon \cdot y^{\prime \prime}+y^{\prime}=\lambda, \\
y(0, \varepsilon)=a_{0}+\lambda, \quad y(1, \varepsilon)=a_{1}+\lambda, \\
\alpha \cdot y(1, \varepsilon)+\beta y^{\prime}(1, \varepsilon)=b+\sigma \lambda .
\end{gathered}
$$

Obviously, the considered problems (82) satisfy all the requirements described in Section 2 . Then, the solution of the problems (82) can be represented in the following form:

$$
y(t, \varepsilon)=\lambda \cdot t+a_{0}+\lambda+\frac{a_{1}-a_{0}}{1-\mathrm{e}^{-1 / \varepsilon}}-\frac{a_{1}-a_{0}}{1-\mathrm{e}^{-1 / \varepsilon}} \cdot \mathrm{e}^{-\mathrm{t} / \varepsilon} .
$$

According to (61) and (62), we formulate the degenerate problem in the following form:

$$
\begin{gathered}
\bar{y}^{\prime}=\lambda, \\
\bar{y}(1)=a_{1}+\lambda, \quad \alpha \cdot \bar{y}(1)+\beta \cdot \bar{y}^{\prime}(1)=b+\sigma \lambda .
\end{gathered}
$$

Then, the pair $\left(\bar{y}\left(t, \lambda_{0}\right), \lambda_{0}\right)$ is the unique solution for the nonhomogeneous boundary value problem (84) on the interval $[0,1]$, where $\lambda_{0}, \bar{y}\left(t, \lambda_{0}\right)$ are expressed as

$$
\bar{y}=\lambda_{0} t+a_{1}, \quad \lambda_{0}=\frac{b-\alpha a_{1}}{\alpha+\beta-\sigma} .
$$

From (83), it follows that the derivative $y^{\prime}(t, \varepsilon)$ at the point $t=0$ has the pole for $\varepsilon$ :

$$
y^{\prime}(0, \varepsilon)=O\left(\frac{1}{\varepsilon}\right)
$$


Hence, $y(t, \varepsilon)$ at the point $t=0$ has the phenomenon of a first-order initial jump, and above all, the magnitude of the initial jump is determined as

$$
\Delta=\lim _{\varepsilon \rightarrow 0} y(0, \varepsilon)-\bar{y}(0)=a_{0}+\lambda_{0}-a_{1}=a_{0}+\lambda_{0}-L_{1} \bar{y} .
$$

(2) The cases of $n-1=m_{1}>m_{2} \geq \cdots \geq m_{n}, n-1=$ $r_{1}>r_{i}$, and $i=\overline{2, n}$ can be researched in accordance with the above-represented scheme.

(3) We can use the same method for researching the asymptotics of the solution of the boundary value problem with a moving boundary:

$$
\begin{gathered}
\varepsilon y^{(n)}+A_{1}(t) y^{(n-1)}+\cdots+A_{n}(t) y=h(t), \\
\sum_{j=0}^{m_{i}} \alpha_{i j} y^{(j)}(0, \varepsilon)+\sum_{j=0}^{r_{i}} \beta_{i j} y^{(j)}(\lambda, \varepsilon)=a_{i}, \quad i=1, \ldots, n+1,
\end{gathered}
$$

where $\lambda$ is an unknown function of $\varepsilon$.

\section{Conflict of Interests}

The author declares that there is no conflict of interests regarding the publication of this paper.

\section{References}

[1] A. N. Tihonov, "On independence of the solutions of differential equations from a small parameter," Matematicheskii Sbornik, vol. 22, no. 2, pp. 193-204, 1948.

[2] A. N. Tihonov, "Systems of differential equations containing small parameters within derivatives," Matematicheskii Sbornik, vol. 31, no. 3, pp. 575-586, 1952.

[3] M. I. Višik and L. A. Lyusternik, "Regular degeneration and boundary layer for linear differential equations with a small parameter," Uspekhi Matematicheskikh Nauk, vol. 12, no. 5, pp. 3-122, 1957.

[4] A. B. Vasilyeva, "Asymptotics of the solutions of some boundary value problems for quasilinear equations within a small parameter and a senior derivative," Doklady Akademii Nauk SSSR, vol. 123, no. 4, pp. 583-586, 1958.

[5] V. F. Butuzov, "Angular boundary layer in the mixed singularly perturbed problems for hyperbolic equations of the second order," Doklady Akademii Nauk SSSR, vol. 235, no. 5, pp. 9971000, 1977.

[6] M. I. Višik and L. A. Lyusternik, "On initial jump for non-linear differential equations containing a small parameter," Doklady Akademii Nauk SSSR, vol. 132, no. 6, pp. 1242-1245, 1960.

[7] K. A. Kasymov, "On asymptotics of the solutions of Cauchy problem with boundary conditions for non-linear ordinary differential equations containing a small parameter," Uspekhi Matematicheskikh Nauk, vol. 17, no. 5, pp. 187-188, 1962.

[8] Z. N. Zhakupov, "Asymptotic behavior of the solutions of boundary value problem for some class of non-linear equation systems containing a small parameter," Izvestiya Akademii Nauk Kazakhskoĭ SSR, no. 5, pp. 42-49, 1971.
[9] D. N. Nurgabylov, "Asymptotic expansion of the solution of a boundary value problem with internal initial jump for nonlinear systems of differential equations," Izvestiya Akademii Nauk Kazakhskoĭ SSR, no. 3, pp. 62-65, 1984.

[10] Yu. I. Neimmark and V. N. Smirnova, "Singularly perturbed problems and the Painlevé paradox," Differentsial'niye Uravneniya, vol. 36, no. 11, pp. 1493-1500, 2000.

[11] V. F. Butuzov and A. B. Vasiliyeva, "On asymptotics of the solution of a contrast structure type," Matematicheskie Zametki, vol. 42, no. 6, pp. 881-841, 1987.

[12] A. V. Kibenko and A. M. Perov, "On a two-point boundaryvalue problem with a parameter," Akademiya Nauk Ukrainskő̆ SSR, no. 10, pp. 1259-1265, 1961.

[13] D. S. Dzhumabayev, "On the unique solvability of the linear point-to-point problems with a parameter," Izvestiya Akademii Nauk SSSR, no. 1, pp. 31-37, 1999.

[14] L. Schlesinger, "Über asymptotische darstellungen der lösungen linearer differentialsysteme als funktionen eines parameters," Mathematische Annalen, vol. 63, no. 3, pp. 277-300, 1907.

[15] G. D. Birkhoff, "On the asymptotic character of the solutions of certain linear differential equations containing a parameter," Transactions of the American Mathematical Society, vol. 9, no. 2, pp. 219-231, 1908.

[16] S. A. Lomov, Introduction to the General Theory of Singular Perturbations, Nauka, Moscow, Russia, 1981.

[17] K. A. Kasymov and D. N. Nurgabyl, "Asymptotic estimates for the solution of a singularly perturbed boundary value problem with an initial jump for linear differential equations," Differential Equations, vol. 40, no. 5, pp. 641-651, 2004. 


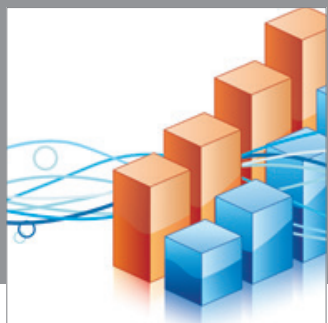

Advances in

Operations Research

mansans

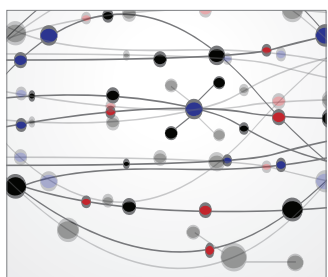

The Scientific World Journal
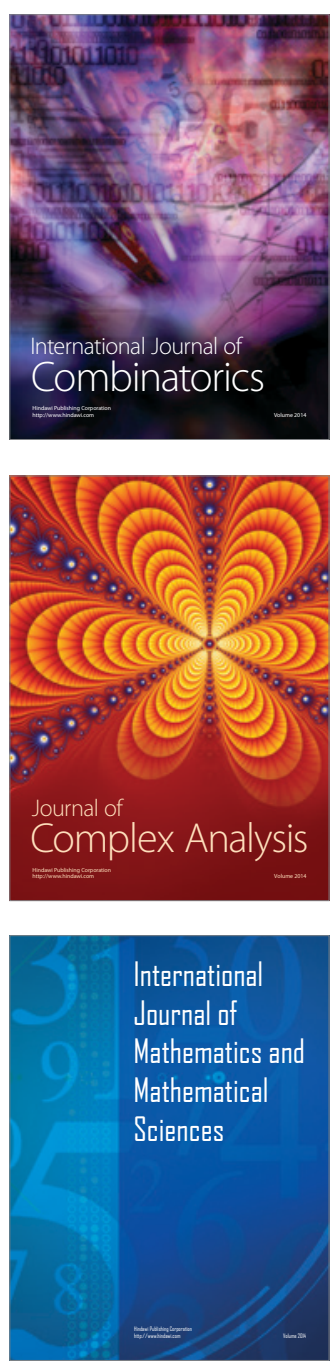
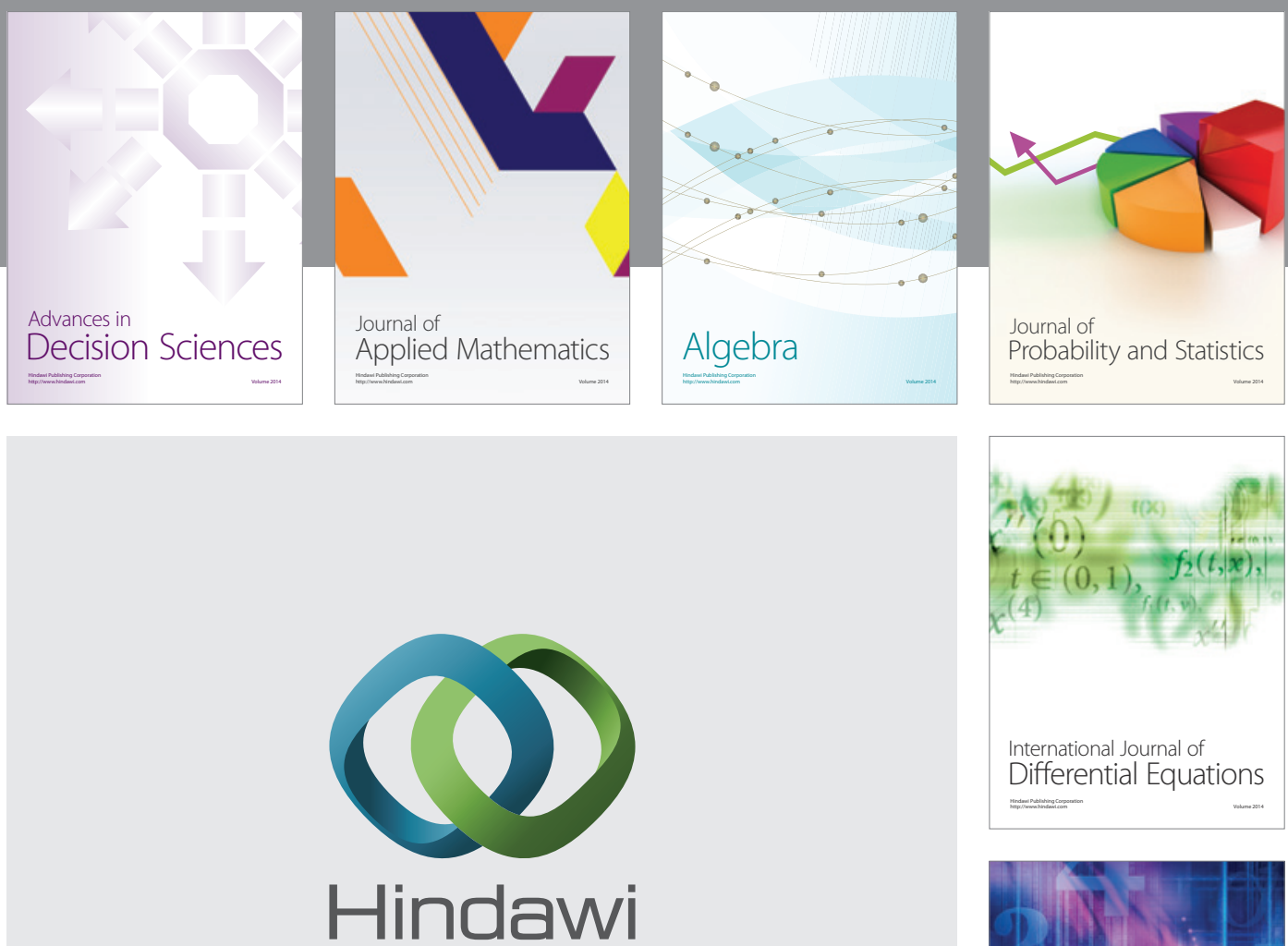

Submit your manuscripts at http://www.hindawi.com
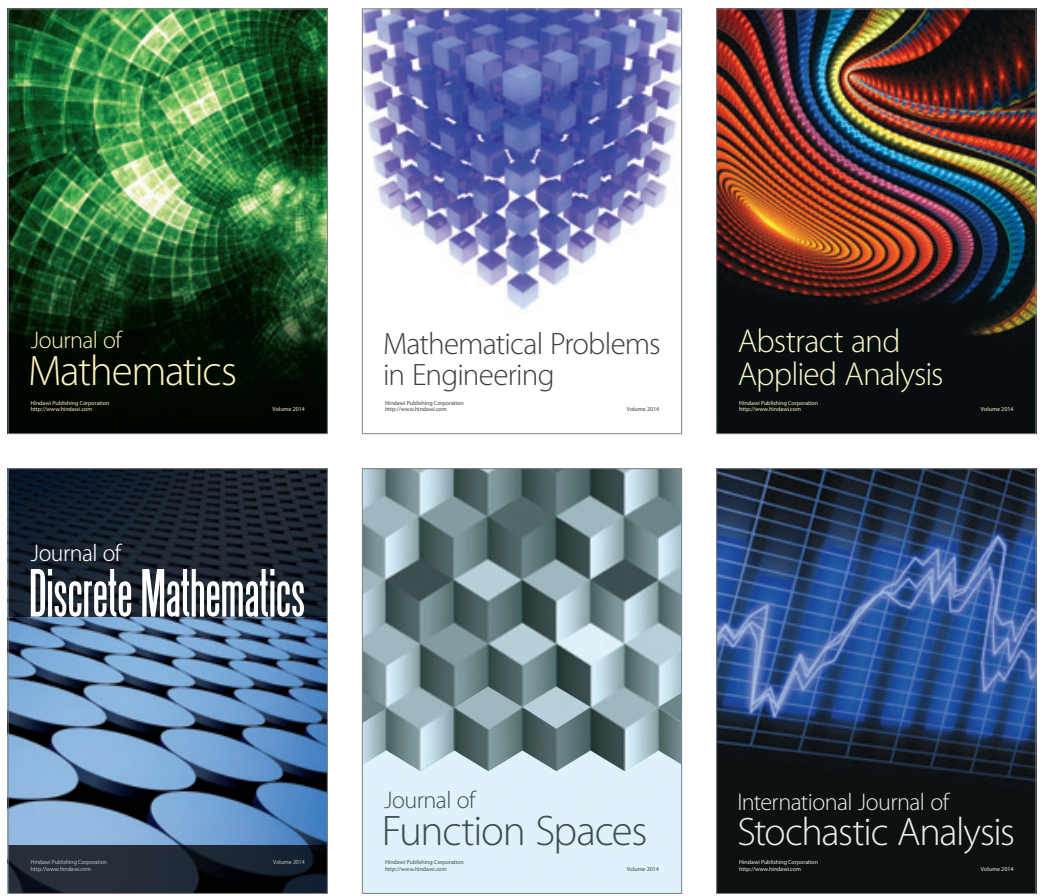

Journal of

Function Spaces

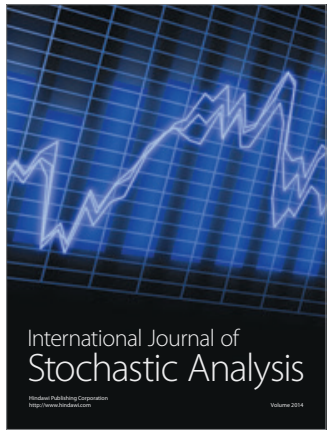

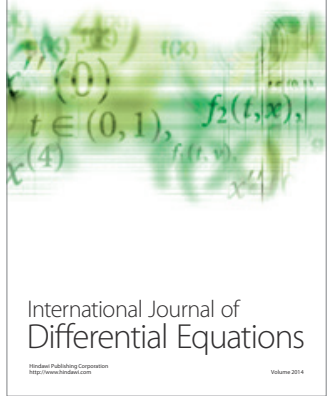
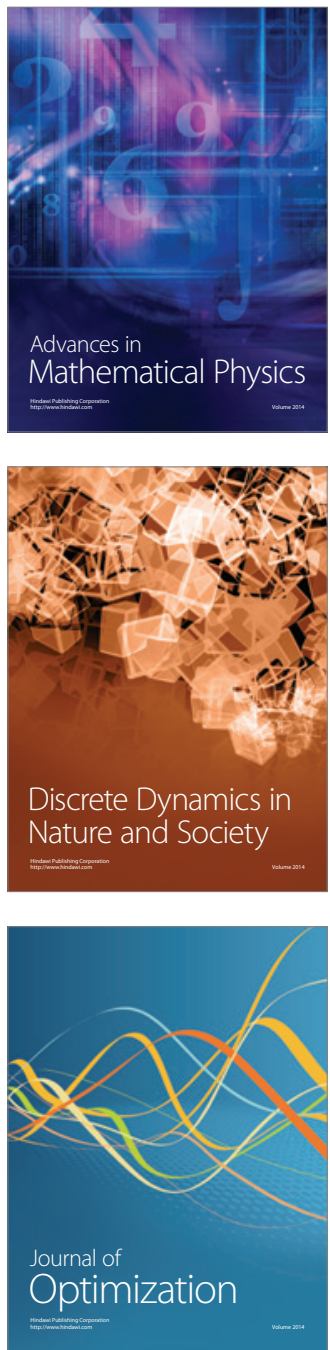